\title{
Exploration of Clinician Adherence and Competency as Predictors of Treatment Outcomes in a School-Based Homework and Organization Intervention for Students with ADHD
}

\author{
Rosanna Breaux, Ph.D. ${ }^{1}$, https://orcid.org/0000-0001-5500-6950 \\ Kelcie D. Willis, M.S. ${ }^{2}$, https://orcid.org/0000-0002-3974-634X \\ Hana-May Eadeh, M.A. ${ }^{3}$, https://orcid.org/0000-0002-4916-0828 \\ Elizaveta Bourchtein, Ph.D. ${ }^{2}$, https://orcid.org/0000-0002-1898-6985 \\ Alissa McCowan, B.S. ${ }^{2}$ \\ Joshua M. Langberg, Ph.D. ${ }^{2}$, https://orcid.org/0000-0003-0169-2793 \\ ${ }^{1}$ Virginia Polytechnic Institute and State University, Department of Psychology; \\ ${ }^{2}$ Virginia Commonwealth University, Department of Psychology \\ ${ }^{3}$ University of Iowa, Department of Psychological and Brain Sciences
}

Correspondence concerning this article should be addressed to Rosanna Breaux, $\mathrm{PhD}$, Virginia Tech, Child Study Center, Department of Psychology, 460 Turner St. NW, Suite 207, Blacksburg, VA 24060, rbreaux@vt.edu, 540-231-8511

Funding: This study was supported by the Institute of Education Sciences, U.S. Department of Education, through Grant R305A130011 to Virginia Commonwealth University and the senior author. The opinions expressed are those of the authors and do not represent views of the Institute or the U.S. Department of Education.

Acknowledgements: We would like to thank the families, teachers, graduate students, project coordinators, and school clinicians who made this research possible.

Conflict of Interest: The authors have no conflict of interest to disclose.

Availability of data and material: Data available upon request from the senior author.

This is the post-print version of the manuscript accepted in School Mental Health:

Breaux, R., Willis, K. D., Eadeh, H. M., Bourchtein, E., McCowan, A., \& Langberg, J. M. (2021). Exploration of Clinician Adherence and Competency as Predictors of Treatment Outcomes in a School-Based Homework and Organization Intervention for Students with ADHD. School Mental Health, 13(2), 406-419. https://doi.org/10.1007/s12310-021-09430-0 


\begin{abstract}
Addressing the complex homework and organization problems faced by students with attentiondeficit/hyperactivity disorder (ADHD) must be balanced with ensuring that interventions are feasible to implement. The Homework, Organization, and Planning Skills (HOPS) intervention was developed to be a brief intervention implemented in typical school settings with minimal training/support required for school clinicians. Given this, it is critical to explore how clinician adherence and competency relate to student outcomes. Participants included 107 adolescents with ADHD (Mage $=11.95, S D=1.04 ; 69 \%$ male; $57 \%$ on ADHD medication) who participated in the HOPS intervention. Path analyses examined the relation between clinician adherence (fidelity to session content, session length) and competency (e.g., empathic, enthusiastic, calm, collaborative), controlling for baseline scores of the outcome measure and other relevant therapeutic processes (parent and adolescent engagement, working alliance). Clinician fidelity and competency were inversely related, such that highly competent clinicians had lower fidelity to the HOPS content. Importantly, clinician competency was a significant predictor of fewer parent-reported adolescent organizational problems and a higher percentage of assignments turned in post-HOPS, whereas clinician fidelity was unrelated to treatment outcomes. Session length was inversely related to parent-reported homework performance, such that longer session length was associated with worse homework outcomes. Findings suggest that school clinician competency is an important factor in predicting positive treatment outcomes for youth who participate in school-based homework and organization interventions. Training to help school clinicians improve their competency including being more empathetic, calm, motivating, and collaborative with students, and to help them flexibly implement school-based interventions is warranted.
\end{abstract}

Keywords: school mental health; ADHD; adolescents; engagement; fidelity 
Students with attention-deficit/hyperactivity disorder (ADHD) experience significant problems recording homework assignments, managing homework materials, and completing homework (e.g., Boyer et al., 2015; DuPaul \& Langberg, 2014). These difficulties with organization and homework completion contribute to lower school grades and achievement scores as well as higher rates of school dropout in comparison to their peers without ADHD (DuPaul \& Stoner, 2003; Frazier et al., 2007; Langberg et al., 2011). Homework and organization difficulties are especially apparent during middle school, when there is an increase in the number of teachers, student independence, and workload (Evans et al., 2005a; Evans et al., 2005b). Interventions have been developed for use in clinic and school settings to address homework and organizational difficulties among youth with ADHD (e.g., Abikoff et al., 2013; Evans et al., 2016; Pfiffner et al., 2013; Sibley et al., 2016). These interventions typically train multiple skills, such as materials organization (e.g., binder, bookbag), accurate and consistent homework recording, and planning and time-management skills. Further, most interventions actively engage parents and teach them how to monitor and reinforce skill implementation.

The Homework, Organization, and Planning Skills (HOPS) intervention was developed to be a brief, skills-based treatment implemented in typical school settings by school mental health providers (hereafter referred to as school clinicians; Langberg et al., 2018). Specifically, the HOPS intervention was developed to address the research-to-practice gap caused by existing ADHD interventions being primarily designed for implementation in clinic settings and requiring substantial training and supervision for clinicians (see Langberg et al., 2018). The HOPS intervention was designed to be brief and implemented during the school day, requiring limited time, resources, and training (see Langberg et al., 2018). The HOPS intervention includes 16 individual sessions delivered during the school day (e.g., during elective periods) and two parent sessions held in the evenings at the school. The individual sessions occur twice weekly for the first 10 sessions and weekly for the final six sessions, resulting in an 11-week intervention. Individual sessions with the adolescent are expected to be approximately 20 minutes each; parent sessions are expected to be approximately 1 hour each.

Although considerable research has examined the efficacy of homework and organization interventions for youth with ADHD (see Bikic et al., 2017 for review), and predictors of treatment response (e.g., Breaux et al., 2019; Langberg et al., 2016b), limited research has examined the role of therapeutic processes (i.e., the interactions and communications between clinicians and their clients; Orlinsky et al., 2015) in treatment response. The handful of studies that have evaluated the role of therapeutic processes have largely focused on the working alliance (i.e., the bond between the client and the clinician and the ability of the clinician to work with the client in a collaborative manner on therapeutic tasks and treatment goals; Breaux et al., 2018; Langberg et al., 2013, 2016b) and parent and youth engagement and adherence (Breaux et al., 2018; Clarke et al., 2015; Rooney et al., 2018; Villodas et al., 2014). Most of these studies have found parent engagement/adherence and the working alliance to be important in predicting treatment outcomes (Clarke et al., 2015; Langberg et al., 2013; Villodas et al., 2014), whereas attendance has been largely unrelated to treatment outcomes (Breaux et al., 2018; Clarke et al., 2015). However, results have not been fully consistent, with one study finding that parent adherence did not predict treatment outcomes (Rooney et al., 2018). Although adolescent engagement has been linked to treatment outcomes for the HOPS intervention, the relation was inverse in nature, (i.e., youth who were rated as more engaged had poorer treatment outcomes), suggesting that students who are more impaired academically may be more engaged in sessions (Breaux et al., 2018). The heterogeneous findings of prior research may have resulted from 
differences in intervention format (i.e., individual homework-focused interventions; Breaux et al., 2018; Langberg et al., 2013 vs. multicomponent interventions that include skills groups and classroom interventions; Rooney et al., 2018). Overall, the evidence accumulated to date suggests that parent and youth active engagement and adherence with treatment and the bond clinicians have with families improve intervention response. Further, these therapeutic processes are relevant for treatment outcomes among interventions for children (Rooney et al., 2018; Villodas et al., 2014), adolescents (Breaux et al., 2018; Langberg et al., 2013, 2016b), and parents of youth with ADHD (Clarke et al., 2015; Rooney et al., 2018).

In the broader clinical child intervention research literature, three categories of therapeutic processes have been proposed to affect treatment outcomes: relational factors (working alliance, involvement/engagement); therapeutic interventions (adherence, differentiation), and therapist competence (skillfulness, responsiveness; McLeod et al., 2013). Thus, prior research in homework and organizational interventions for students with ADHD have only examined aspects of two of these processes: relational factors (working alliance and parent/youth involvement and engagement) and therapeutic interventions (parent adherence). To our knowledge, no study to date has examined therapist competence or clinician aspects of therapeutic interventions in predicting treatment outcomes of youth with ADHD who complete school-based homework and organization interventions. Importantly, in the larger treatment outcome literature, there is evidence that the role of clinician adherence and competency is significantly decreased or no longer significant when you control for the working alliance (Webb et al., 2010), highlighting the importance of examining the impact of these factors above and beyond other therapeutic processes.

Given this backdrop, the present study sought to explore the role of clinician adherence (fidelity to session content and total session length) and clinician competency (Vocal Animation and Excitement, Vocal Relaxation, Empathy, Collaborative Relationship, and Positivity/Encouragement/Motivation) in the treatment outcomes of adolescents with ADHD who participated in the HOPS intervention. These conceptualizations are consistent with prior research, which views adherence as the extent to which clinicians conform to intervention protocol (Hogue et al., 2008), and competence as a clinician's personal- and process-level skills (Forgatch et al., 2005; Perepletchikova \& Kazdin, 2005; Stein et al., 2007). Specifically, previous research classified competency as the clinician's ability to communicate with, effectively deliver information to, and respond to the participants' needs (Breitenstein et al., 2010). Importantly, we examined these associations above and beyond other therapeutic processes that have been related to treatment outcomes among youth receiving HOPS and other homework and organization skills interventions (i.e., working alliance, parent and adolescent engagement). As such, this study sought to answer the following research question:

Do clinician adherence and clinician competency predict treatment outcomes for adolescents with ADHD who participate in the HOPS intervention, after accounting for other relevant therapeutic processes, demographic variables, and baseline functioning? It was hypothesized that both clinician adherence and clinician competency would be positively related to parent- and teacher-reported homework performance and the percentage of homework assignments turned in, and negatively associated with parent- and teacher-reported organizational problems, controlling for other therapeutic processes (i.e., the relational factors of the working alliance, parent engagement, adolescent engagement), key demographic variables 
(e.g., adolescent sex, family income, medication status) ${ }^{1}$, and baseline functioning (i.e., baseline scores for each outcome). Demographic variables were explored as potential covariates, given prior research highlighting the role of social determinants and demographic factors for HOPS and other homework and organization interventions for students with ADHD (e.g., Evans et al., 2016; Green et al., 2020).

\section{Participants}

\section{Method}

Participants were 107 middle-school students ( 74 boys, 33 girls; $M_{\text {age }}=11.95$ years, $S D=$ 1.04) with ADHD who received the HOPS intervention as part of a randomized controlled trial (Langberg et al., 2018) ${ }^{2}$. Participants were recruited from seven public middle schools in a suburban area of the Southeastern United States, representing a range of settings and family backgrounds. Participants were racially diverse: 54\% White, 29\% Black, 13\% multiracial, and $4 \%$ another race or did not disclose their race; $9 \%$ identified as Latinx. Median family income was $\$ 62,500$. Six school clinicians who had recently graduated with a Masters' Degree in School Counseling (White females ages $25-27$ years) delivered the intervention. School clinicians were hired and paid through the research grant.

\section{Procedures}

Consistent with traditional school identification and intervention practices, school staff distributed recruitment flyers describing the study (e.g., offering "homework interventions for students with attention and behavioral difficulties and/or with ADD/ADHD”) to parents of students they thought would benefit from the intervention. Interested families completed a phone screen; families whose children displayed at least four of nine ADHD parent-reported inattention symptoms during the phone screen were scheduled for a full inclusion/exclusion evaluation. Inclusion criteria included (a) attended a participating school, (b) met full diagnostic criteria for ADHD based on the parent Children's Interview for Psychiatric Syndromes (ChIPS; Weller et al., 2000) diagnostic interview or the parent interview combined with teacher ratings using the Vanderbilt ADHD Rating Scale, in the event of borderline diagnoses (e.g., endorsement of 5 symptoms of inattention and indication of impairment and high teacher ratings of inattention including the endorsement of unique symptoms from parents; Wolraich et al., 2003), (c) an estimated IQ of at least 80 and (d) no diagnosis of pervasive developmental disorder, bipolar disorder, or psychosis. Participants were allowed to have other comorbidities to be representative of an ADHD sample (38\% oppositional defiant disorder, 31\% anxiety disorder, 5\% depressive disorder). IQ was estimated by the Block Design and Vocabulary subtests of the Wechsler Intelligence Scale for Children - Fourth Edition (Wechsler, 2003); these two subtests have been found to provide a reliable and valid estimate of full scale IQ (see Sattler, 2008). A total of 381 adolescents were referred, 355 families of whom were screened ( 24 did not meet screening criteria; two were not interested), 285 met study criteria (target sample size was 264), and 280 were randomized to one of two intervention groups or to a waitlist control. Only participants randomized to the HOPS intervention group are included in this study, as the second intervention was designed specifically to serve as an active control for the HOPS intervention in the larger RCT. Of the 111 adolescents who received the HOPS intervention, 108 completed measures

\footnotetext{
${ }^{1}$ Because all the clinicians were newly graduated, White female school counselors, there was not enough clinician diversity in demographic factors to explore these as covariates.

${ }^{2}$ In the larger randomized controlled trial, 113 families were randomized to receive HOPS and 111 started the intervention. Four students did not have recordings for any of their sessions due to one of the school clinicians losing a portion of their audio recordings.
} 
post-intervention. All 111 participants were included in the study, as statistical analyses could account for this missing data. For more information on inclusion and exclusion criteria, participant demographics, and the larger RCT see Langberg et al. (2018).

When this study began in 2013, no established observational method for measuring clinical adherence and competence for more general skills-based behavioral interventions existed; instead, the field largely relied on clinician self-report. However, a large body of research suggests that clinicians may not be the best reporters of their own abilities (Adams et al., 1999; Creed et al., 2016; Mathieson et al., 2009). As such, a coding system was developed to assess school clinician fidelity and competency. Specifically, in the present study clinician adherence was measured by clinician fidelity to session content (proportion of expected content covered each session) and total session length (across all adolescent and parent sessions). Clinician competency was measured as an average across ratings on five general therapeutic skills based on those that have been used in other clinician competency research (e.g., Blackburn et al., 2001; Breitenstein et al., 2010): vocal animation and excitement; vocal relaxation; empathy; collaborative relationship; positivity, encouragement, and motivation.

School clinicians audio-recorded the intervention sessions. The coding system for clinician adherence and competency was applied to the seven intervention sessions where new skills were introduced ${ }^{3}$, sessions 3-4, 6-9, and 12. Each week, coders (2 advanced undergraduate students and 1 clinical psychology graduate student) met with the first author and other coders to discuss discrepancies in clinician fidelity and adherence. Practice tapes were used until all raters gained reliability on the coding system (agreement for all codes $>.70$ ). Once coders became reliable, they began coding HOPS participants with $20 \%$ of tapes being rated by two independent coders. Weekly meetings were held to review double coded tapes; double coded tapes were interspersed with individually coded tapes to ensure reliability was maintained. When any discrepancies were present for fidelity items and when discrepancies $>1$ were present for competency items, the same two coders listened to the session(s) where the discrepancies occurred and came to a consensus regarding the discrepant codes. For the $20 \%$ of tapes that were double coded, consensus codes were used. Intra-class correlations (ICCs) were calculated to determine inter-rater reliability (Hallgren, 2012).

\section{Clinician Adherence}

Session Length. The length of each session was coded from the audio-recordings. The average adolescent session lasted 17.42 minutes $(S D=3.50)$, with a total number of minutes across all 16 adolescent sessions ranging from 110 to 385 minutes. The expected session length for HOPS adolescent content would be 320 minutes (20 minutes for each of 16 sessions). The average parent session lasted 42.15 minutes $(S D=14.26)$, with the total number of minutes for parent sessions ranging from 0 to 133 minutes, where 0 represents families that did not attend either parent session. The expected session length for HOPS parent content would be 120 minutes ( 2 meetings, each for 1 hour). In the present study, session length, one aspect of clinician

\footnotetext{
${ }^{3}$ The HOPS fidelity checklists contain a core set of items that assess behaviors that providers implement every session (e.g., completion of checklists, administering points). When new skills were taught and practiced, the checklists were expanded to include additional items. In coding and reviewing the items that were present across all sessions, it quickly became clear that the core set of assessment items consistently happened every session, resulting in little variability in these items. This is not surprising, as HOPS was designed to be highly feasible for providers to implement with minimal training. Therefore, to reduce coder burden, we focused on the sessions that also included teaching and practice of new skills, which captures both the core assessment items (e.g., checklist completion) and the additional items focused on teaching and practicing new skills. Doing so also allowed us to assess fidelity to delivery pace - that is, whether skills were implemented on the timeline specified in the manual.
} 
adherence, was a sum of the total number of minutes for both adolescent and parent sessions.

Clinician Fidelity. Clinician fidelity, an aspect of clinician adherence, was assessed by the independent coders using a checklist of six to nine items, where the number of items depended on the particular goals of that session (e.g., Did the counselor ask the student if there were any questions regarding homework recording?, Did the counselor review the Materials Organization Plan?). Coders used these checklist items to determine if the school clinician abided by the HOPS treatment manual, including tasks such as reviewing previously introduced skills, introducing new skills at the correct time point, and asking the participant if they had any questions. For all fidelity items, the coders responded using a dichotomous "yes" or "no." Clinician fidelity was then calculated to be the proportion of items that were covered in each session, averaged across sessions. The ICC for inter-rater reliability of clinician fidelity was .79.

\section{Clinician Competency}

Clinician competency assessed how well the school clinician delivered the treatment content and responded to the participant throughout the session. Coders rated the school clinician using a 7-point Likert scale in five general therapeutic skills: (1) Vocal Animation and Excitement, (2) Vocal Relaxation, (3) Empathy, (4) Collaborative Relationship, and (5) Positivity/Encouragement/Motivation. Vocal Animation and Excitement described the school clinician's variety in tempo, pitch, volume, and emotion when delivering content; higher scores were awarded when the school clinician sounded more animated and excited, whereas lower scores reflect a more monotone or inexpressive style. Vocal Relaxation described how tense/anxious the school clinician sounded throughout the session; higher scores were given when the school clinician seemed calm and relaxed, while lower scores were given when the school clinician seemed tense or anxious with a tone that was tight, shaky, or nervous. Empathy described the school clinician's use of emotional reflection and ability to respond in a supportive manner to negative statements; higher scores were awarded when the school clinician responded in an understanding and empathetic manner, whereas lower scores were given when the school clinician was not responsive or dismissive to the negative comments. Collaborative Relationship described the school clinician's ability to engage the student in treatment through the use of open-ended questions and eliciting the adolescent's input; higher sores were given when the school clinician sought student input and student-generated ideas, whereas lower scores were given when the school clinician directed the session with little to no student input. Lastly, Positivity/Encouraging/Motivation described the school clinician's ability to "cheerlead" the student during treatment; higher scores were awarded when the school clinician was positive, encouraging, and motivating (e.g., "awesome job," "I know you can do it") throughout the session, while lower scores were given in the absence of these behaviors. Given that codes were based on audio recordings, all codes were rated based on the pitch and quality of vocalization as well as the actual statements being made. These five therapeutic skills and the use of a 7-point Likert scale are consistent with the types of behaviors rated (e.g., understanding, interpersonal effectiveness, collaboration) by clinicians in training and supervisors, the format of self- and supervisor-reported measures (see Mathieson et al., 2009), and existing expert-rated scales for cognitive-behavioral therapy (Blackburn et al., 2001). For data reduction purposes and because competency items were highly correlated $(r s=.64-.83)$, we averaged the five competency items into a single clinician competency variable. The ICC for inter-rater reliability of competency was .73 (ICCs ranged from .70 - .78 for individual items). Additionally, internal consistency across sessions was acceptable to very good for each aspect of clinician competency: Vocal Animation and Excitement $=.91$, Vocal Relaxation $=.86$, Empathy $=.67$, Collaborative Relationship $=.79$, 
and Positivity/Encouragement/Motivation $=.71$.

\section{Other Therapeutic Process Measures}

Working Alliance. Working alliance between the adolescent and the school clinician was assessed by school clinician report on the Working Alliance Inventory - Short Revised (Hatcher \& Gillaspy, 2006), a 12-item measure assessing three aspects of the working alliance: agreement on therapeutic tasks, agreement on therapeutic goals, and client-clinician bond. Each item was rated on a 7-point Likert scale ranging from 1 (never) to 7 (always). Scores on the Working Alliance Inventory have been reported as reliable $(\alpha \mathrm{s}=.85-.92)$ and demonstrate convergent validity with other alliance instruments (Hatcher \& Gillaspy, 2006). In the present study, internal consistency was .92. Working alliance was included as a control variable.

Adolescent Engagement. At the end of each session, school clinicians rated how engaged the adolescent was on a 7-point Likert scale ranging from 1 (not engaged, monotone, blank affect) to 7 (high engaged, animated, enthusiastic). Average adolescent engagement across sessions was included as a control variable.

Parent Engagement. At the end of each parent meeting, school clinicians rated how engaged the parent was during each parent meeting on a 7-point Likert scale from 1 (not engaged, monotone, blank affect) to 7 (high engaged, animated, enthusiastic). Average parent engagement across sessions was included as a control variable.

\section{Outcome Measures}

Homework Performance Questionnaire (HPQ). Parents and teachers separately completed the HPQ (Power et al., 2007), which consists of 13 items on a 5-point scale, Parents and teachers answer each item by choosing the percentage that corresponds to the amount of time a certain behavior occurs (e.g., student writes down homework assignments independently or manages homework time well). Items were worded in the positive so that $90 \%$ to $100 \%$ of the time indicates that the adolescent does that behavior consistently well. Scores on the HPQ have demonstrated convergent validity with other measures of homework problems (Power et al., 2007, 2015). Internal consistency was $\alpha=.91$ and .94 for parents and $\alpha=.96$ and .97 for teachers at baseline and post-intervention, respectively.

Children's Organizational Skills Scale (COSS). The COSS (Abikoff \& Gallagher, 2008) is a measure of organization, planning, and time management skills — consisting of three subscales, Task Planning, Organized Actions, and Memory and Materials Management - and a total score that includes all of the items. Test-retest reliability with the three COSS subscales is high for the parent (.94 - .99) and teacher (.88 - .93) versions (Abikoff \& Gallagher, 2008). The COSS has been validated for use within an ADHD sample (Molitor et al., 2017). In the present study, the COSS total score was used and had adequate internal consistency at both time points (parent $\alpha \mathrm{s}=.80$ at both baseline and post-intervention; teacher $\alpha \mathrm{s}=.84$ and .82 at baseline and post-intervention, respectively).

Assignments Turned In. Teachers reported the percentage of assignments (0\% to 100\%) students turned in on time. This item is similar to one used in the Classroom Performance Survey (Brady, Evans, Berlin, Bunford, \& Kern, 2012), which has been used to track homework completion in multiple studies (e.g., Langberg et al., 2016a; Meyer \& Kelley, 2007). Scores on this item has demonstrated good clinical utility in distinguishing teacher identified academically impaired students from non-impaired students. Moreover, this measure displays convergent validity with other measures of academic impairment (Brady et al., 2012).

\section{Demographic Variables}

Participant age was reported by parents on a demographic form, and ranged from 10-15 
years old. Parents reported on adolescent biological sex $(0=$ male, $1=$ female $)$. Parent education was reported by parents in the number of years of education they received. Parent education in the present study ranged from 6 to 18 years, with $77 \%$ of the sample having a high school education (12 years) or more. Parents reported on family income on a demographic form, and ranged from $\$ 5,000$ to $\$ 225,000$ in the present study. Participants identified as White, Black, BiRacial/Multi-Racial, or another race. In the present study, a dichotomized race variable $(0=$ White, $58 \% ; 1=$ other race, $42 \%$ ) was calculated. Medication status was reported by parents pretreatment $(0=$ not on medication for ADHD, $1=$ on medication for ADHD). Single parent status was coded such that $0=$ single parent and $1=$ two parent household.

\section{Analytic Plan}

Descriptive statistics and correlations between study variables were first examined, including exploring whether key demographic variables were related to any study variables. One-way ANOVAs were run to explore clinician and school differences in clinician competency and adherence variables and between other therapeutic processes that were included as control variables (working alliance, parent and adolescent engagement). Next, a series of regression analyses (Byrne, 2013) were conducted in Mplus version 8 (Muthén and Muthén 1998-2017). These analyses evaluated the role of clinician adherence and competency variables in predicting post-treatment outcomes, controlling for baseline scores for outcome measures, other relevant therapeutic processes (i.e., working alliance, parent and adolescent engagement), and relevant demographic variables. Clinician fidelity and adolescent and parent session length were set to correlate in the model, as it was theorized that clinicians with higher fidelity would be more likely to have longer sessions with parents and adolescents, as they would need more time to cover all the recommended content. Full information maximum likelihood was used to address missing data, which uses all observed information to estimate parameters. Standardized coefficients are presented in figures; standardized coefficients can be used to gauge relative importance of paths and interpreted as $r$-values (Durlak, 2009) with values greater than 10 indicating a small effect, values greater than .30 indicating a medium effect, and values greater than .50 indicating a large effect (Cohen, 1988). Model fit was examined using several indices: Comparative Fit Index (CFI), the root mean square error approximation (RMSEA), and the Standardized Root Mean Square Residual (SRMR). CFI values greater than 90 suggest good fit. RMSEA values less than .06 indicate good fit, values less than .10 indicate adequate fit, and values greater than .10 indicate poor fit. SRMR values less than 0.08 suggest good fit. To control for nesting effects, we used the sandwich variance estimator (Diggle, Heagerty, Liang, \& Zeger, 2002), which produces corrected standard errors in the presence of non-independent data due to nested data structures, in this case, adolescents nested within school clinicians and schools. Posthoc power analyses suggest that models with eight predictors would be adequately powered (i.e., power $=.80$ ) to detect medium and large effects, whereas models with nine, 10 , or 11 predictors would be slightly underpowered (power $=.77, .75$, and .72 , respectively) to detect medium effects. Given the number of analyses being run, alpha was set at .01; however, to balance the risk of possible Type II Error given the power associated with our small sample size, we discuss any values between .01 and .05 as marginally significant.

\section{Preliminary Analyses}

\section{Results}

Descriptive statistics and correlations between therapeutic process variables are presented in Table 1. Interestingly, clinician fidelity and competence were inversely related such that clinicians with higher rated competency had lower fidelity in administering the HOPS 
intervention on average. The two measures of clinician adherence, clinician fidelity and session length were not significantly correlated, nor were the measures of clinician adherence and competency with any of the other therapeutic process variables (working alliance, parent and adolescent engagement). One-way ANOVAs comparing clinicians on adherence and competency measures suggested that clinicians did not significantly differ on fidelity or competency $(F=1.05$ and $0.17, p=.38$ and .96 , respectively), but did significantly differ on total session length $(F=4.74, p=.002)$, supporting the use of sandwich estimation in main analyses to account for nesting within clinicians and schools. Similarly, clinicians did not significantly differ on working alliance $(F=0.91, p=.46)$, but did significantly differ on adolescent and parent engagement $(F=7.44$ and $3.27, p<.001$ and $p=.02$, respectively).

Correlations between key demographic variables (age, sex, parent education, family income, single parent status, race, ethnicity, medication status) and clinician/intervention variables were examined to determine which demographic variables to include as covariates. Age, parent education, ethnicity, and race were unrelated to all study variables and thus were not considered in analyses $(r s=-.22-.17 ; p s>.06)$. Adolescent sex was significantly positively correlated with teacher-reported HPQ $(r=.29, p<.01)$ and parent- and teacher-reported COSS $(r=.42$ and .24 , respectively; $p s<.02$ ), suggesting that females had better homework performance, but worse organization problems. Family income and single parent status were significantly positively correlated with teacher-reported HPQ ( $r=.31$ and $.21, p=.004$ and .048 , respectively) and assignments turned in ( $r=.33$ and $.22, p=.001$ and .032 , respectively), suggesting that adolescents from families with higher incomes and with the parents together had better homework performance and turned in more assignments. As such, adolescent sex, single parent status, and family income were included in the teacher-reported HPQ model; adolescent sex was included in the parent- and teacher-reported COSS models; and single parent status and family income were included in the assignments turned in model.

\section{Role of Clinician Adherence and Competency in Parent-Reported Outcomes}

Results for the regression model examining the relation between clinician adherence and competency in parent-reported homework performance post-HOPS can be found in Figure 1. This model demonstrated acceptable fit: $\mathrm{RMSEA}=0.08, \mathrm{CFI}=0.86$, and $\mathrm{SRMR}=0.08$. Consistent with past research, a stronger working alliance was positively and moderately associated with parent-reported homework performance $(\beta=.39, p<.01)$. Results for the regression model examining the relation between clinician adherence and competency in parent-reported organization problems can be found in Figure 2. This model demonstrated acceptable fit: $\mathrm{RMSEA}=0.09, \mathrm{CFI}=0.86$, and $\mathrm{SRMR}=0.09$. Clinician competency was a marginally significant unique predictor of parent-reported organizational difficulties controlling for other therapeutic processes, adolescent sex, and baseline homework performance $(\beta=-.40, p=.03)$. Specifically, a negative, moderate association was found such that higher clinician competency was associated with less organizational difficulties.

\section{Role of Clinician Adherence and Competency in Teacher-Reported Outcomes}

Results for the regression model examining the relation between clinician adherence and competency in teacher-reported homework performance post-HOPS can be found in Figure 3. This model demonstrated acceptable fit: RMSEA =0.09, CFI=0.91, and SRMR=0.08. Clinician adherence and competency were not unique predictors of teacher-reported homework performance. Results for the regression model examining the relation between clinician adherence and competency in teacher-reported organization problems can be found in Figure 4. This model demonstrated acceptable fit: RMSEA=0.09, CFI=0.92, and SRMR=0.09. Clinician 
adherence and competency were not unique predictors of teacher-reported organizational difficulties. Consistent with past research, a stronger working alliance was negatively and moderately associated with teacher-reported organizational problems, such that stronger working alliances were associated with less teacher-reported organizational problems $(\beta=-.40 p<.01)$. Role of Clinician Adherence and Competency in Percentage of Assignments Turned In

Results for the regression model examining the relation between clinician adherence and competency in the percentage of assignments turned in can be found in Figure 5. This model demonstrated acceptable fit: $\mathrm{RMSEA}=0.09, \mathrm{CFI}=0.91$, and $\mathrm{SRMR}=0.09$. Clinician competency was a marginally significant unique predictor of the percentage of assignments turned in postHOPS, controlling for other therapeutic processes, and baseline percentage of assignments turned in $(\beta=.30, p=.01)$. Specifically, clinicians who were rated as more competent worked with adolescents who turned in a more homework assignments post-intervention.

\section{Discussion}

The present study adds to the limited, but growing, body of research examining the role of therapeutic processes in the treatment outcomes of youth with ADHD who participate in homework and organization interventions (Breaux et al., 2018; Clarke et al., 2015; Langberg et al., 2013, 2016b; Rooney et al., 2018; Villodaset al., 2014). This study is novel in its focus on clinician adherence and competency, and the associated effects of these variables in treatment outcomes above and beyond other relevant therapeutic processes (working alliance, parent and adolescent engagement). Specifically, it is the first to examine the therapeutic competence factor of therapeutic processes along with clinician adherence, an aspect of the therapeutic interventions factor, as described by McLeod et al. (2013) for homework and organization interventions for youth with ADHD. Results highlight that school clinicians who are rated as highly competent may be less likely to adhere to session content, but that this lack of fidelity is not necessarily detrimental to treatment outcomes. Ensuring that school clinicians have high levels of competency (e.g., empathy, enthusiasm, ability to foster a collaborative and motivating relationship) and are able to foster a strong working alliance with students appear to be important for treatment outcomes.

Clinician fidelity (i.e., how well the clinician adhered to expected session content) and clinician competency (i.e., how relaxed, excited, empathic, encouraging, and collaborative the clinician was) were inversely related, such that clinicians who were rated as more competent displayed less fidelity to HOPS session content. Importantly, clinician competency-but not clinician fidelity - was related to treatment outcomes. Taken together, these results suggest that more competent clinicians may be more comfortable adapting session content to meet the individual needs of students, and this flexibility may actually enhance rather than undermine treatment outcomes. For example, a highly competent clinician is more student-directed and collaborative, likely resulting in them recognizing when a student needs additional time to review previously taught material prior to introducing new session content, which would have resulted in a lower fidelity score due to them not covering the expected content during that session. This finding is largely consistent with Stiles and Shapiro's (1994) "responsiveness theory" which suggests that adherence is not an important predictor of treatment outcomes because the optimal frequency and the nature of an intervention should not be determined in advance, and that therapeutic techniques may need to vary from the prescribed manual to be congruent with the needs of the client. Additionally, it fits with the larger therapeutic processes literature, which has found clinician competency, but not clinician adherence to be a unique predictor of treatment outcomes (e.g., Rounsaville et al., 1988; Shaw et al., 1999). However, this 
literature is somewhat mixed, with some studies (e.g., Barber et al., 1996; Hogue et al., 2008) failing to find clinician competency as a significant predictor of treatment outcomes. Finally, our finding is consistent with work with other clinical child and adolescent population (e.g., Coping Cat for anxiety) which has highlighted the importance of flexibility and creativity to individualize manual-based treatments (Kendall et al., 1998).

Our finding that clinician competency is moderately associated with better parent-rated and objective (i.e., percentage of assignments turned in) homework performance measures extends prior research with student-clinician working alliance. Specifically, a strong working alliance has similarly shown to be associated with better treatment outcomes in school-based homework and organization interventions (Breaux et al., 2018; Langberg et al., 2013, 2016b). Surprisingly, clinician competency and working alliance were not significantly correlated in the present study. This may result from when and how working alliance was measured. That is, working alliance was just measured once in the middle of the HOPS intervention. There is some evidence that including multiple alliance measurements starting early in treatment may produce a more accurate estimate of working alliance (Crits-Christoph et al., 2011). As such, it will be important for future research to assess working alliance throughout school-based interventions. Despite this, our results suggest that school clinician competency is a unique predictor of treatment outcomes above and beyond the influence of the working alliance, which was also found to be a unique predictor of parent-reported homework performance and teacher-reported organizational problems in the present study. Together, these findings support common factors research (e.g., Karver et al., 2005, Wampold, 2015) suggesting that 'common factors' like the therapeutic relationship and the empathic, collaborative, and facilitative abilities of the clinician are important for treatment outcomes.

Both parent and adolescent session length were unrelated to treatment outcomes. Additionally, at the bivariate level, session length was unrelated to clinician fidelity and clinician competency, suggesting that longer sessions do not necessarily mean that the clinicians who spent more time in session on average were more likely to adhere to session content or to be more competent (e.g., you could be a poor counselor who speeds through material or you could be a poor counselor who takes longer because you have trouble delivering the material). This will be an important area for future research to continue to explore.

\section{Limitations}

Findings from the present study should be interpreted within the context of several limitations. First, our assessment of clinician competency and adherence was coded based on audio recordings which do not provide as much insight into the actual behaviors that the clinician and adolescent were engaging in; in particular, non-verbal behaviors from the clinician could provide insight into clinician competency (e.g., empathy, engagement). Second, the measure of clinician competency was developed for this study based upon prior studies of self-reported and expert-rated clinician competency (Blackburn et al., 2001; Mathieson et al., 2009), but this measure has not been validated with other intervention samples. Third, several of the control variable measures used in the present study were single items (parent and adolescent engagement) and based solely on a single clinician's report, preventing establishment of interrater reliability. It will be important for future research to look at engagement in a more in-depth manner, and to tease apart the aspects of engagement (e.g., skill utilization during the week) that might be particularly influential, given that neither parent nor adolescent engagement were unique predictors of treatment outcomes in the present study. Relatedly, we examined the control variables of working alliance and adolescent engagement separately, but they are likely related 
constructs that influence each other over time. Similarly, both of these constructs are also related to our measure of clinician competence. Examination of how the working alliance, adolescent engagement, and clinician competence influence each other and change throughout the intervention is an important area for future research. Fourth, we only measured working alliance using clinician-rated alliance; given prior research suggesting differential findings for adolescent versus clinician working alliance (e.g., Breaux et al., 2018), it will be important for future work to also include adolescent report and to examine how these different perspectives may relate to treatment outcomes. Fifth, all of our models were underpowered to detect small effects, and our models that controlled for adolescent sex and/or family income were somewhat underpowered to detect medium effects (power $=.77$ and .75 , respectively). As such, it will be important for future work to replicate these analyses with a larger sample. Sixth, although our recruitment procedures were designed to mirror traditional school identification and intervention practices, it is possible that these procedures may still limit generalizability of our findings, as there may be biases in which families agree to engage in research and completed the inclusion/exclusion evaluation (e.g., parents with a higher education). Finally, this study included only six school clinicians, all of whom were newly graduated, White female school counselors who were paid by the study, which could limit the generalizability of the findings and precluded us from examining clinician demographic factors as covariates.

\section{Conclusions}

The results of this study have important implications for school clinician-delivered interventions. Most school-based intervention protocols focus largely on how to deliver the core content of the treatment; however, our results suggest that clinician fidelity to intervention content is unrelated to treatment outcomes. Instead, results suggest that it is more important to work with school clinicians on developing their competency in general therapeutic skills - that is, how to be enthusiastic, empathetic, collaborative, motivating, and calm during sessions —and to provide guidance and examples of how to develop a strong bond with students and parents (i.e., how to develop a strong working alliance). School counselors rarely receive such training in therapeutic processes (Lever et al., 2014), which may limit the efficacy of school-based interventions. Additionally, results suggest that encouraging clinicians to implement interventions flexibly based on individual student needs may actually lead to better outcomes. This is an important area for future work to continue to explore. Findings from this study need to be replicated, but preliminarily suggest that trainings for skills-based homework and organizational intervention protocols should emphasize development of clinician competency, fostering a strong bond with the student, and collaboratively establishing treatment goals, rather than focusing on clinicians adhering to the specific intervention protocol. 


\section{References}

Abikoff, H., \& Gallagher, R. (2008). Assessment and remediation of organizational skills deficits in children with ADHD. In K. McBurnett \& L. Pfiffner (Eds), Attention deficit hyperactivity disorder: Concepts, controversies and new directions (pp. 137-152). Taylor \& Francis Group.

Abikoff, H., Gallagher, R., Wells, K. C., Murray, D. W., Huang, L., Lu, F., \& Petkova, E. (2013). Remediating organizational functioning in children with ADHD: Immediate and long-term effects from a randomized controlled trial. Journal of Consulting and Clinical Psychology, 81, 113-128. https://doi.org/10.1037/a0029648

Adams, A. S., Soumerai, S. B., Lomas, J., \& Ross-Degnan, D. (1999). Evidence of self-report bias in assessing adherence to guidelines. International Journal for Quality in Health Care, 11, 187-192. https://doi.org/10.1093/intqhc/11.3.187

Barber, J. P., Crits-Christoph, P., \& Luborsky, L. (1996). Effects of therapist adherence and competence on patient outcome in brief dynamic therapy. Journal of Consulting and Clinical Psychology, 64(3), 619-622. https://doi.org/10.1037/0022-006X.64.3.619

Bikic, A., Reichow, B., McCauley, S. A., Ibrahim, K., \& Sukhodolsky, D. G. (2017). Metaanalysis of organizational skills interventions for children and adolescents with attentiondeficit/hyperactivity disorder. Clinical Psychology Review, 52, 108-123. https://doi.org/10.1016/j.cpr.2016.12.004

Blackburn, I. M., James, I. A., Milne, D. L., Baker, C., Standart, S., Garland, A., \& Reichelt, F. K. (2001). The revised cognitive therapy scale (CTS-R): psychometric properties. Behavioural and Cognitive Psychotherapy, 29, 431-446. http://dx.doi.org/10.1017/S1352465801004040

Boyer, B. E., Geurts, H. M., Prins, P. J., \& Van der Oord, S. (2015). Two novel CBTs for adolescents with ADHD: The value of planning skills. European Child \& Adolescent Psychiatry, 24, 1075-1090. https://doi.org/10.1007/s00787-014-0661-5

Brady, C.E., Evans, S.W., Berlin, K.S., Bunford, N., \& Kern, L. (2012) Evaluating school impairment with adolescents using the classroom performance survey, School Psychology Review, 41, 429-444. https://doi.org/10.1080/02796015.2012.12087498

Breaux, R. P., Langberg, J. M., Bourchtein, E., Eadeh, H. M., Molitor, S. J., \& Smith, Z. R. (2019). Brief homework intervention for adolescents with ADHD: Trajectories and predictors of response. School Psychology, 34, 201-211. https://doi.org/10.1037/spq0000287

Breaux, R. P., Langberg, J. M., McLeod, B. D., Molitor, S. J., Smith, Z. R., Bourchtein, E., \& Green, C. D. (2018). The importance of therapeutic processes in school-based psychosocial treatment of homework problems in adolescents with ADHD. Journal of Consulting and Clinical Psychology, 86, 427-438. http://dx.doi.org/10.1037/ccp0000300

Breitenstein, S. M., Gross, D., Garvey, C. A., Hill, C., Fogg, L., \& Resnick, B. (2010). Implementation fidelity in community-based interventions. Research in Nursing \& Health, 33, 164-173. https://doi.org/10.1002/nur.20373

Byrne, B. M. (2013). Structural equation modeling with Mplus: Basic concepts, applications, and programming. Routledge.

Cohen, J. (1988). Statistical power analyses for the social sciences. Lawrence Erlbauni Associates.

Creed, T. A., Wolk, C. B., Feinberg, B., Evans, A. C., \& Beck, A. T. (2016). Beyond the label: 
Relationship between community therapists' self-report of a cognitive behavioral therapy orientation and observed skills. Administration and Policy in Mental Health and Mental Health Services Research, 43(1), 36-43. https://doi.org/10.1007/s10488-014-0618-5

Crits-Christoph, P., Gibbons, M. B., Hamilton, J., Ring-Kurtz, S., \& Gallop, R. (2011). The dependability of alliance assessments: The alliance-outcome correlation is larger than you might think. Journal of Consulting and Clinical Psychology, 79, 267-278. https://doi.org/10.1037/a0023668

Diggle, P., Diggle, P. J., Heagerty, P., Liang, K. Y., Heagerty, P. J., \& Zeger, S. (2002). Analysis of longitudinal data. Oxford University Press.

DuPaul, G. J., \& Langberg, J. M. (2014). Educational impairments in children with ADHD. In R. A. Barkley (Ed.), Attention-deficit/hyperactivity disorder: A handbook for diagnosis and treatment (4th ed.). Guilford.

DuPaul, G. J. and Stoner, G. 2003. ADHD in the schools. Guilford.

Durlak, J. A. (2009). How to select, calculate, and interpret effect sizes. Journal of Pediatric Psychology, 34, 917-928. https://doi.org/10.1093/jpepsy/jsp004

Evans, S. W., Langberg, J., Raggi, V., Allen, J., \& Buvinger, E. C. (2005a). Development of a school-based treatment program for middle school youth with ADHD. Journal of Attention Disorders, 9, 343-353. https://doi.org/10.1177/1087054705279305

Evans, S. W., Langberg, J. M., Schultz, B. K., Vaughn, A., Altaye, M., Marshall, S. A., \& Zoromski, A. K. (2016). Evaluation of a school-based treatment program for young adolescents with ADHD. Journal of Consulting and Clinical Psychology, 84, 15-30. https://doi.org/10.1037/ccp0000057

Evans, S. W., Serpell, Z., \& White, C. (2005b). The transition to middle school: Preparing for challenge and success. Attention! Magazine (CHADD), 29-31.

Forgatch, M.S., Patterson, G.R., \& DeGarmo, D.S. (2005). Evaluating fidelity: Predictive validity for a measure of competent adherence to the Oregon model of parent management training. Behavior Therapy, 36, 3-13. https://doi.org/10.1016/S00057894(05)80049-8

Frazier, T. W., Youngstrom, E. A., Glutting, J. J., \& Watkins, M. W. (2007). ADHD and achievement: Meta-analysis of the child, adolescent, and adult literatures and a concomitant study with college students. Journal of Learning Disabilities, 40, 49-65. https://doi.org/10.1177/00222194070400010401

Green, C. D., Dvorsky, M. R., Langberg, J. M., Jones, H. A., \& Floyd, A. L. (2020). The Impact of Social Determinants of Health on the Efficacy of School-Based Interventions for Adolescents with ADHD. School Mental Health, Advanced Online Publication. https://doi.org/10.1007/s12310-020-09367-w

Hallgren, K. A. (2012). Computing inter-rater reliability for observational data: an overview and tutorial. Tutorials in Quantitative Methods for Psychology, 8(1), 23-34. https://doi.org/10.20982/tqmp.08.1.p023

Hatcher, R. L., \& Gillaspy, J. A. (2006). Development and validation of a revised short version of the Working Alliance Inventory. Psychotherapy Research, 16, 12-25. https://doi.org/10.1080/10503300500352500

Hogue, A., Henderson, C.E., Dauber, S., Barajas, P.C., Fried, A., \& Liddle, H.A. (2008). Treatment adherence, competence, and outcome in individual and family therapy for adolescent behavior problems. Journal of Consulting and Clinical Psychology, 76, 544555. https://doi.org/10.1037/0022-006X.76.4.544 
Karver, M. S., Handelsman, J. B., Fields, S., \& Bickman, L. (2005). A theoretical model of common process factors in youth and family therapy. Mental Health Services Research, 7, 35-51. https://doi.org/10.1007/s11020-005-1964-4

Kendall, P. C., Chu, B., Gifford, A., Hayes, C., \& Nauta, M. (1998). Breathing life into a manual: Flexibility and creativity with manual-based treatments. Cognitive and Behavioral Practice, 5(2), 177-198. https://doi.org/10.1016/S1077-7229(98)80004-7

Langberg, J. M., Becker, S. P., Epstein, J. N., Vaughn, A. J., \& Girio-Herrera, E. (2013). Predictors of response and mechanisms of change in an organizational skills intervention for students with ADHD. Journal of Child and Family Studies, 22, 1000-1012. https://doi.org/10.1007/s10826-012-9662-5

Langberg, J. M., Dvorsky, M. R., Molitor, S. J., Bourchtein, E., Eddy, L. D., Smith, Z. R., ... \& Eadeh, H. M. (2018). Overcoming the research-to-practice gap: A randomized trial with two brief homework and organization interventions for students with ADHD as implemented by school mental health providers. Journal of Consulting and Clinical Psychology, 86, 39-55. https://doi.org/10.1037/ccp0000265

Langberg, J. M., Dvorsky, M. R., Molitor, S. J., Bourchtein, E., Eddy, L. D., Smith, Z., ... \& Evans, S. W. (2016a). Longitudinal evaluation of the importance of homework assignment completion for the academic performance of middle school students with ADHD. Journal of School Psychology, 55, 27-38. https://doi.org/10.1016/j.jsp.2015.12.004

Langberg, J. M., Evans, S. W., Schultz, B. K., Becker, S. P., Altaye, M., \& Girio-Herrera, E. (2016b). Trajectories and predictors of response to the Challenging Horizons Program for adolescents with ADHD. Behavior Therapy, 47, 339-354. https://doi.org/10.1016/j.beth.2016.01.001

Langberg, J. M., Molina, B. S., Arnold, L. E., Epstein, J. N., Altaye, M., Hinshaw, S. P., ... \& Hechtman, L. (2011). Patterns and predictors of adolescent academic achievement and performance in a sample of children with attention-deficit/hyperactivity disorder. Journal of Clinical Child \& Adolescent Psychology, 40, 519-531. https://doi.org/10.1080/15374416.2011.581620

Lever N.A., Lindsey M., O’Brennan L., Weist M.D. (2014) Preservice Training for School Mental Health Clinicians. In: Weist M., Lever N., Bradshaw C., Owens J. (eds) In Handbook of School Mental Health (pp. 45-58). Springer.Mathieson, F. M., Barnfield, T., \& Beaumont, G. (2009). Are we as good as we think we are? Self-assessment versus other forms of assessment of competence in psychotherapy. Cognitive Behaviour Therapist, 2, 43-50. https://doi.org/10.1017/S1754470X08000081

Meyer, K., \& Kelley, M. L. (2007). Improving homework in adolescents with attentiondeficit/hyperactivity disorder: Self vs. parent monitoring of homework behavior and study skills. Child \& Family Behavior Therapy, 29, 25-42. https://doi.org/10.1300/J019v29n04_02

McLeod, B. D., Southam-Gerow, M. A., Tully, C. B., Rodriguez, A., \& Smith, M. M. (2013). Making a case for treatment integrity as a psychosocial treatment quality indicator for youth mental health care. Clinical Psychology: Science and Practice, 20(1), 14-32. https://doi.org/10.1111/cpsp.12020

Molitor, S. J., Langberg, J. M., Evans, S. W., Dvorsky, M. R., Bourchtein, E., Eddy, L. D., ... \& Oddo, L. E. (2017). Evaluating the factor validity of the Children's Organizational Skills Scale in Youth with ADHD. School Mental Health, 9, 143-156. 
https://doi.org/10.1007/s12310-016-9205-0

Muthén, L.K. and Muthén, B.O. (1998-2017). Mplus User's Guide. Eighth Edition. Muthén \& Muthén.

Orlinsky, D., Heinonen, E., Hartmann, A. (2015). Psychotherapy process research. In: J. D. Wright (Ed.), International Encyclopedia of the Social \& Behavioral Sciences, $2^{\text {nd }}$ Edition (pp. 515-520). Elsevier.

Perepletchikova, F., \& Kazdin, A.E. (2005). Treatment integrity and therapeutic change: Issues and research recommendations. Clinical Psychology: Science and Practice, 12, 365-383. https://doi.org/10.1093/clipsy.bpi045

Power, T. J., Dombrowski, S. C., Watkins, M. W., Mautone, J. A., \& Eagle, J. W. (2007). Assessing children's homework performance: Development of multi-dimensional, multiinformant rating scales. Journal of School Psychology, 45, 333-348. https://doi.org/10.1016/j.jsp.2007.02.002

Power, T. J., Watkins, M. W., Mautone, J. A., Walcott, C. M., Coutts, M. J., \& Sheridan, S. M. (2015). Examining the validity of the Homework Performance Questionnaire: Multiinformant assessment in elementary and middle school. School Psychology Quarterly, 30, 260-275. http://dx.doi.org/10.1037/spq0000081

Pfiffner, L. J., Villodas, M., Kaiser, N., Rooney, M., \& McBurnett, K. (2013). Educational outcomes of a collaborative school-home behavioral intervention for ADHD. School Psychology Quarterly, 28, 25-36. https://doi.org/10.1037/spq0000016

Rooney, M., Hinshaw, S., McBurnett, K., \& Pfiffner, L. (2018). Parent adherence in two behavioral treatment strategies for the predominantly inattentive presentation of ADHD. Journal of Clinical Child \& Adolescent Psychology, 47(sup1), S233-S241. https://doi.org/10.1080/15374416.2016.1236341

Rounsaville, B.J., O’Malley, S., Foley, S., Weissman, M.M. (1988). Role of manual-guided training in the conduct and efficacy of interpersonal psychotherapy for depression. Journal of Consulting and Clinical Psychology, 56, 681-688. https://doi.org/10.1037/0022-006X.56.5.681

Sattler, J. M. (2008). Assessment of children: Cognitive foundations. JM Sattler.

Shaw, B. F., Elkin, I., Yamaguchi, J., Olmsted, M., Vallis, T. M., Dobson, K. S., Lowery, A., Sotsky, S. M., Watkins, J. T., \& Imber, S. D. (1999). Therapist competence ratings in relation to clinical outcome in cognitive therapy of depression. Journal of Consulting and Clinical Psychology, 67, 837-846. https://doi.org/10.1037/0022-006X.67.6.837

Sibley, M. H., Graziano, P. A., Kuriyan, A. B., Coxe, S., Pelham, W. E., Rodriguez, L., ... \& Ward, A. (2016). Parent-teen behavior therapy+ motivational interviewing for adolescents with ADHD. Journal of Consulting and Clinical Psychology, 84, 699-712. http://dx.doi.org/10.1037/ccp0000106

Stein, K.F., Sargent, J.T., \& Rafaels, N. (2007). Intervention research: Establishing fidelity of the independent variable in nursing clinical trials. Nursing Research, 56, 54-62.

Villodas, M. T., McBurnett, K., Kaiser, N., Rooney, M., \& Pfiffner, L. J. (2014). Additive effects of parent adherence on social and behavioral outcomes of a collaborative school-home behavioral intervention for ADHD. Child Psychiatry \& Human Development, 45, 348360. https://doi.org/10.1007/s10578-013-0405-7

Wampold, B. E. (2015). How important are the common factors in psychotherapy? An update. World Psychiatry, 14, 270-277. https://doi.org/10.1002/wps.20238

Webb, C. A., DeRubeis, R. J., \& Barber, J. P. (2010). Therapist adherence/competence and 
treatment outcome: A meta-analytic review. Journal of Consulting and Clinical Psychology, 78, 200-211. https://doi.org/10.1037/a0018912

Wechsler, D. (2003). Wechsler intelligence scale for children-WISC-IV. San Antonio, TX: Psychological Corporation.

Wolraich, M. L., Lambert, W., Doffing, M. A., Bickman, L., Simmons, T., \& Worley, K. (2003). Psychometric properties of the Vanderbilt ADHD diagnostic parent rating scale in a referred population. Journal of Pediatric Psychology, 28, 559-568.

https://doi.org/10.1093/jpepsy/jsg046 


\section{Table 1}

Descriptive Statistics of and Correlations Between Therapeutic Process and Outcome Variables

\begin{tabular}{|c|c|c|c|c|c|c|c|c|c|c|c|c|c|}
\hline & $\begin{array}{c}M \\
(S D)\end{array}$ & Range & 1 & 2 & 3 & 4 & 5 & 6 & 7 & 8 & 9 & 10 & 11 \\
\hline 1. Clinician Fidelity & $\begin{array}{c}0.89 \\
(0.06)\end{array}$ & $0.69-1.00$ & -- & & & & & & & & & & \\
\hline 2. Adolescent & 260.32 & $110-386$ & -.003 & -- & & & & & & & & & \\
\hline Session Length & $(57.73)$ & & & & & & & & & & & & \\
\hline 3. Parent Session & 57.04 & $0-133$ & -.030 & .12 & -- & & & & & & & & \\
\hline Length & (30.93) & & & & & & & & & & & & \\
\hline 4. Clinician & 5.69 & $4.20-6.89$ & $-.34^{* * *}$ & .12 & -.07 & -- & & & & & & & \\
\hline Competency & $(0.66)$ & & & & & & & & & & & & \\
\hline 5. Working Alliance & $\begin{array}{r}60.89 \\
(9.34)\end{array}$ & $21-72$ & $.23^{*}$ & -.07 & .04 & .02 & -- & & & & & & \\
\hline $\begin{array}{l}\text { 6. Adolescent } \\
\text { Engagement }\end{array}$ & $\begin{array}{l}6.15 \\
(0.88)\end{array}$ & $3-7$ & -.23 & -.02 & -.09 & .20 & $.41^{* *}$ & -- & & & & & \\
\hline $\begin{array}{l}\text { 7. Parent } \\
\text { Engagement }\end{array}$ & $\begin{array}{l}6.36 \\
(1.01)\end{array}$ & $2-7$ & .18 & -.05 & .00 & -.05 & .10 & .09 & -- & & & & \\
\hline 8. Parent HPQ & $\begin{array}{c}33.95 \\
(11.24)\end{array}$ & $8-52$ & .02 & -.14 & -.10 & .10 & $.37^{* * * *}$ & .17 & .18 & -- & & & \\
\hline 9. Teacher HPQ & $\begin{array}{c}28.85 \\
(12.89)\end{array}$ & $1-52$ & .09 & -.11 & .05 & .07 & $.40^{* * * *}$ & .00 & $.21^{*}$ & $.35^{* *}$ & -- & & \\
\hline 10. Parent COSS & $\begin{array}{c}57.73 \\
(11.01)\end{array}$ & $39-90$ & -.12 & .09 & .09 & -.10 & -.10 & -.03 & $-.22^{*}$ & $-.57^{* * *}$ & -.01 & -- & \\
\hline 11. Teacher COSS & $\begin{array}{l}58.22 \\
(8.92)\end{array}$ & $40-85$ & -.12 & -.05 & .13 & -.06 & $-.31^{* *}$ & .07 & -.10 & $-.36^{* *}$ & $-.66^{* * *}$ & $.37^{* *}$ & -- \\
\hline $\begin{array}{l}\text { 12. Assignments } \\
\text { Turned In }\end{array}$ & $\begin{array}{c}67.59 \\
(24.45)\end{array}$ & $1-100$ & .08 & $-.22^{*}$ & .17 & $.22^{*}$ & $.22^{*}$ & -.12 & $.25^{*}$ & $.39^{* * *}$ & $.68^{* * * *}$ & $-.23^{*}$ & $-.48^{* * * *}$ \\
\hline
\end{tabular}

Note. $\mathrm{HPQ}=$ Homework Performance Questionnaire; COSS = Children's Organizational Skills Scale 


\section{Figure 1}

Role of Clinician Adherence and Competency in Parent-Reported Homework Performance

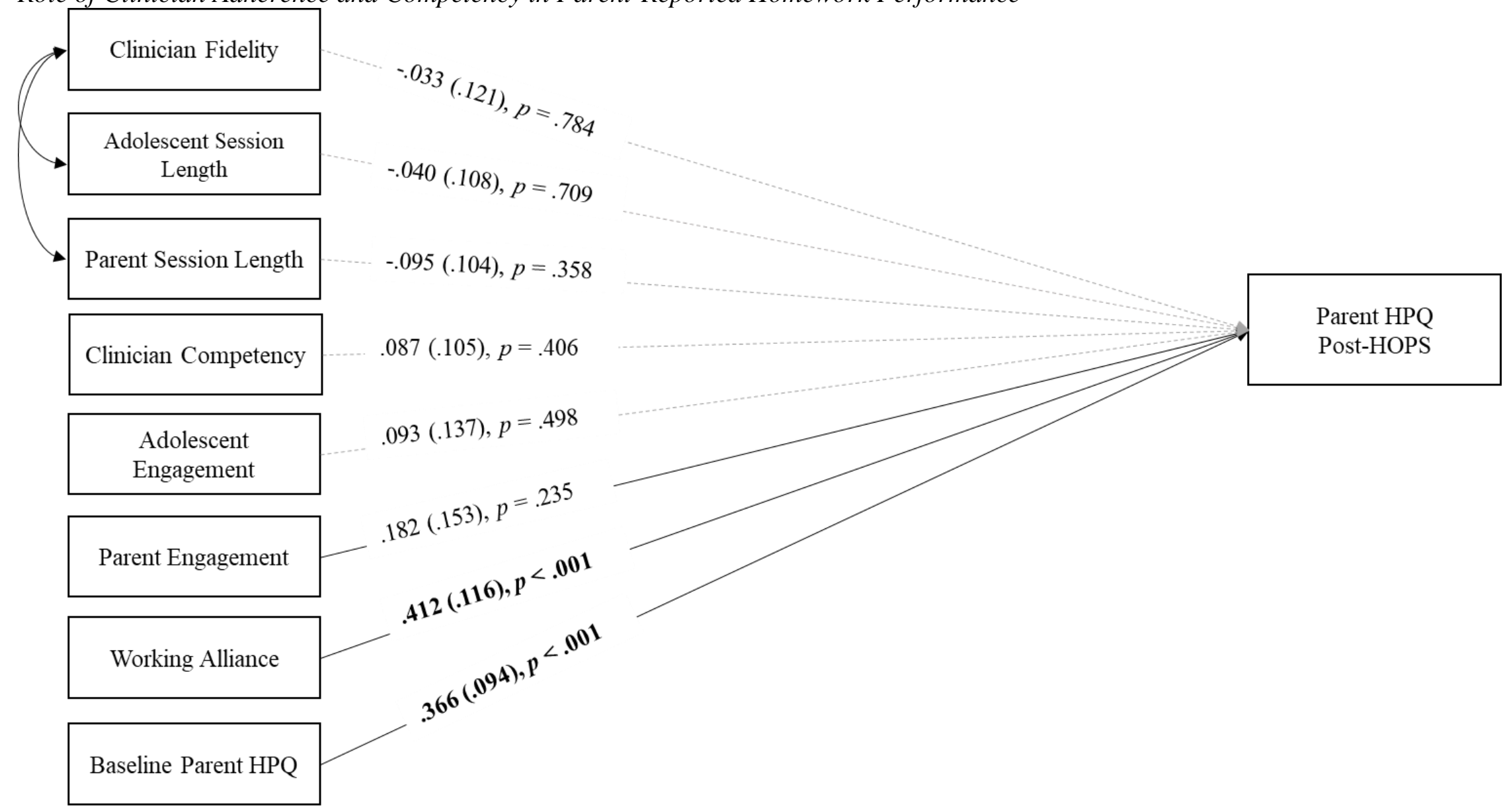

Note. $\mathrm{ADHD}=$ attention-deficit/hyperactivity disorder; $\mathrm{HPQ}=$ Homework Performance Questionnaire; HOPS = Homework, Organization, and Planning Skills. Standardized coefficients presented in figure. Higher scores on the HPQ indicate better homework performance. 


\section{Figure 2}

Role of Clinician Adherence and Competency in Parent-Reported Organization Problems

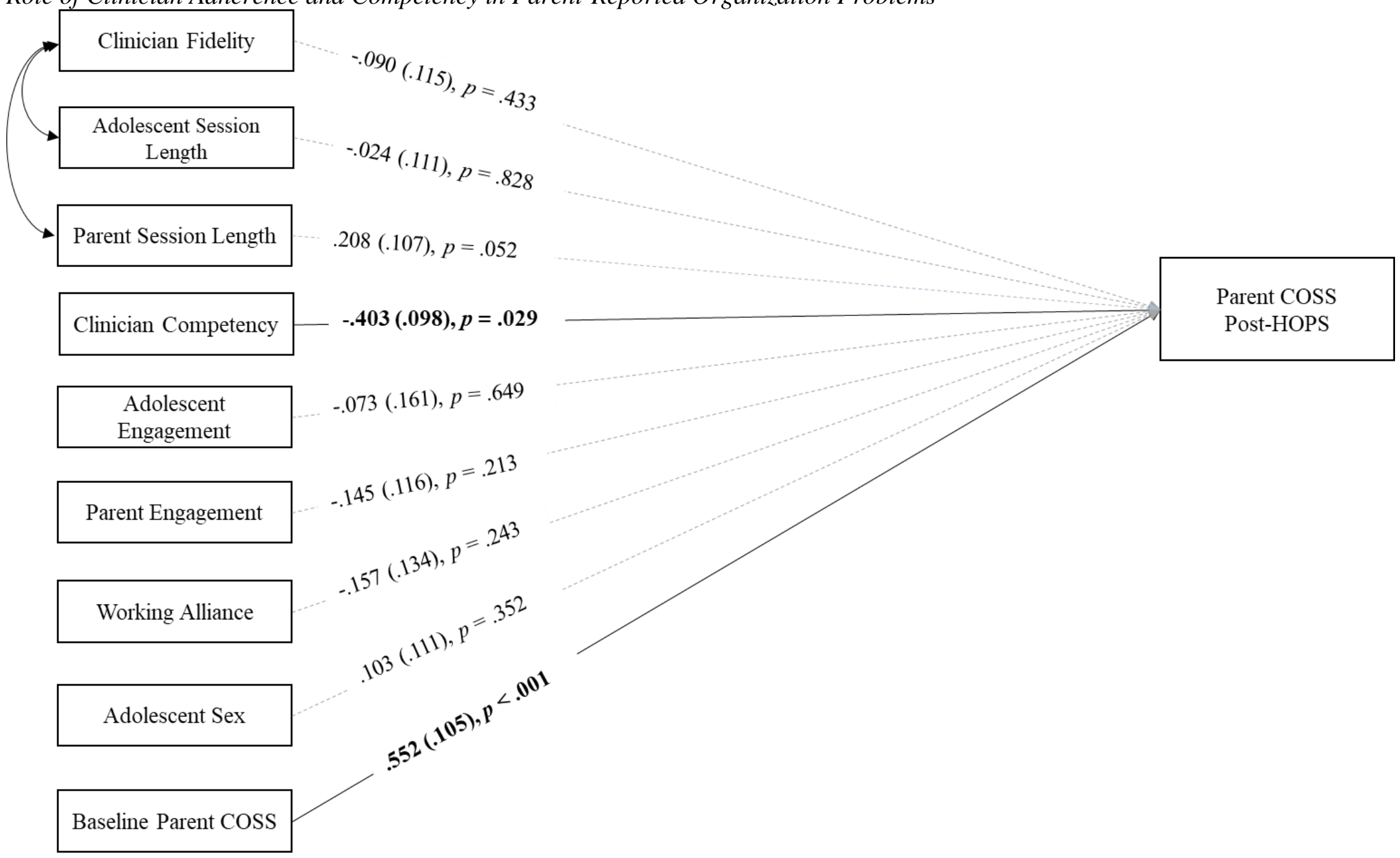

Note. $\mathrm{ADHD}=$ attention-deficit/hyperactivity disorder; $\mathrm{COSS}=$ Children's Organizational Skills Scale; HOPS = Homework, Organization, and Planning Skills. Standardized coefficients presented in figure. Higher scores on the COSS indicate more organization problems. 


\section{Figure 3}

Role of Clinician Adherence and Competency in Teacher-Reported Homework Performance

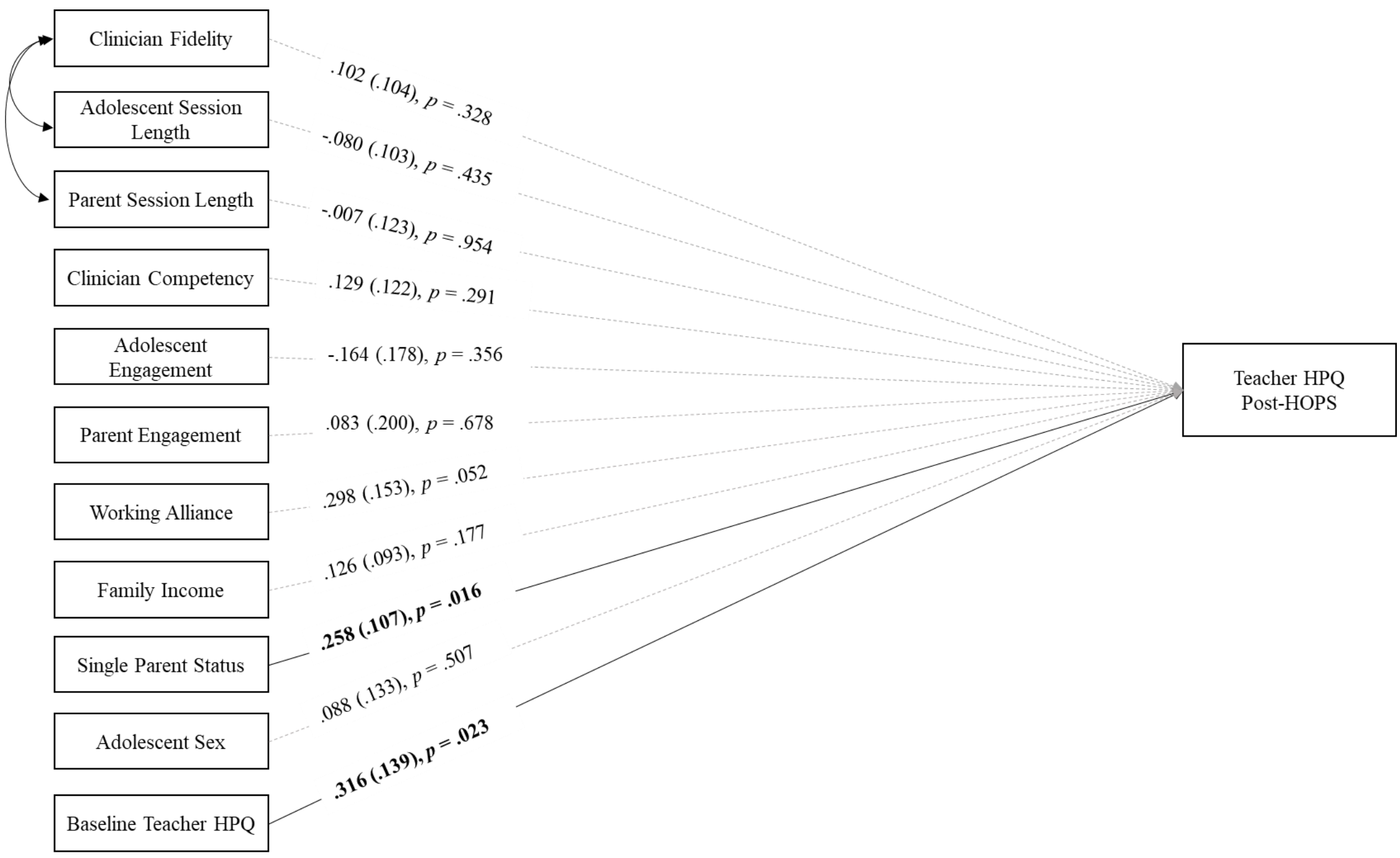

Note. $\mathrm{ADHD}=$ attention-deficit/hyperactivity disorder; HPQ = Homework Performance Questionnaire; HOPS = Homework, Organization, and Planning Skills. Standardized coefficients presented in figure. Higher scores on the HPQ indicate better homework performance. 


\section{Figure 4}

Role of Clinician Adherence and Competency in Teacher-Reported Organization Problems

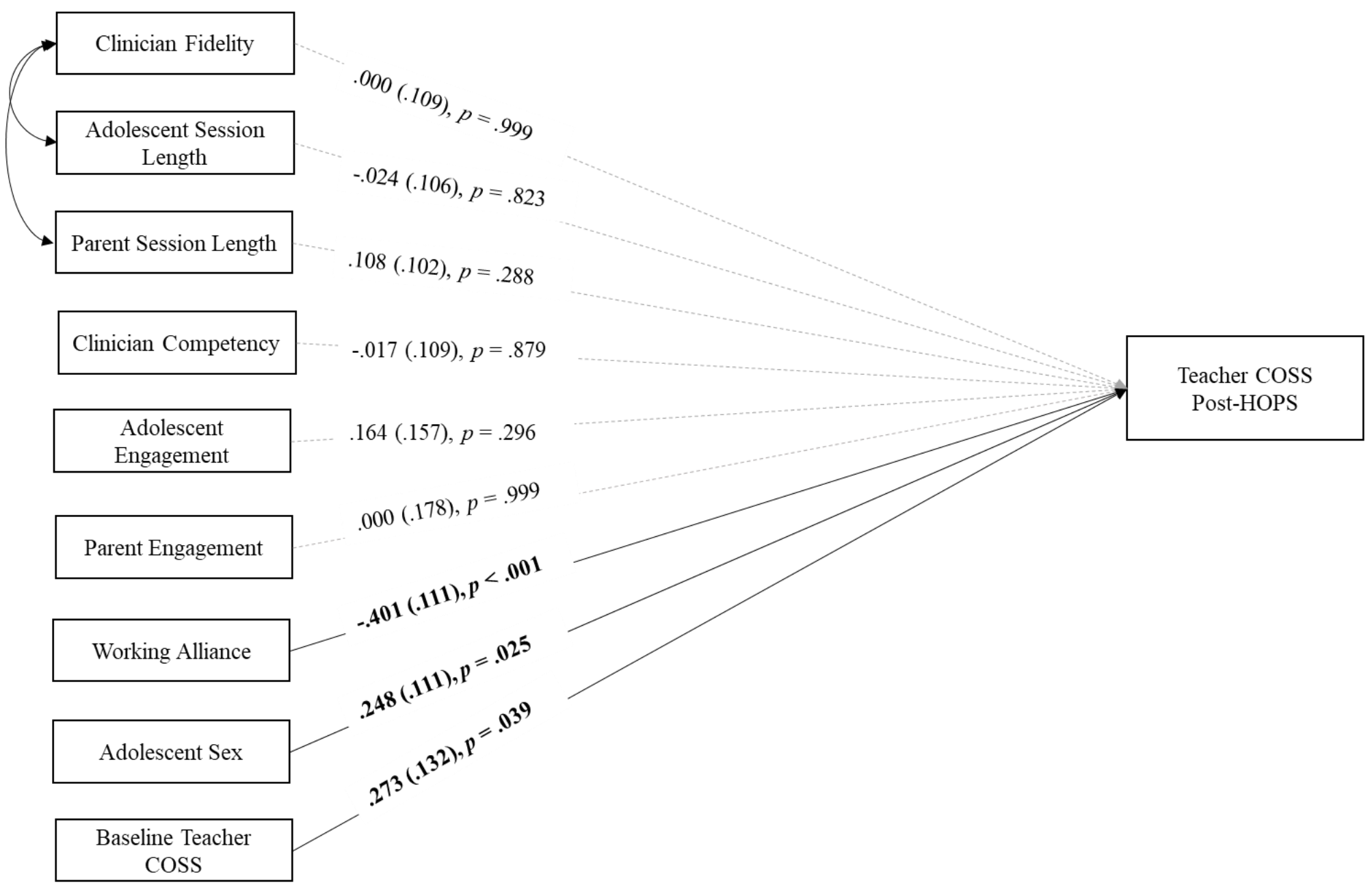

Note. $\mathrm{ADHD}=$ attention-deficit/hyperactivity disorder; $\mathrm{COSS}=$ Children's Organizational Skills Scale; HOPS = Homework, Organization, and Planning Skills. Standardized coefficients presented in figure. Higher scores on the COSS indicate more organization problems. 


\section{Figure 5}

Role of Clinician Adherence and Competency in Percentage of Assignments Turned In

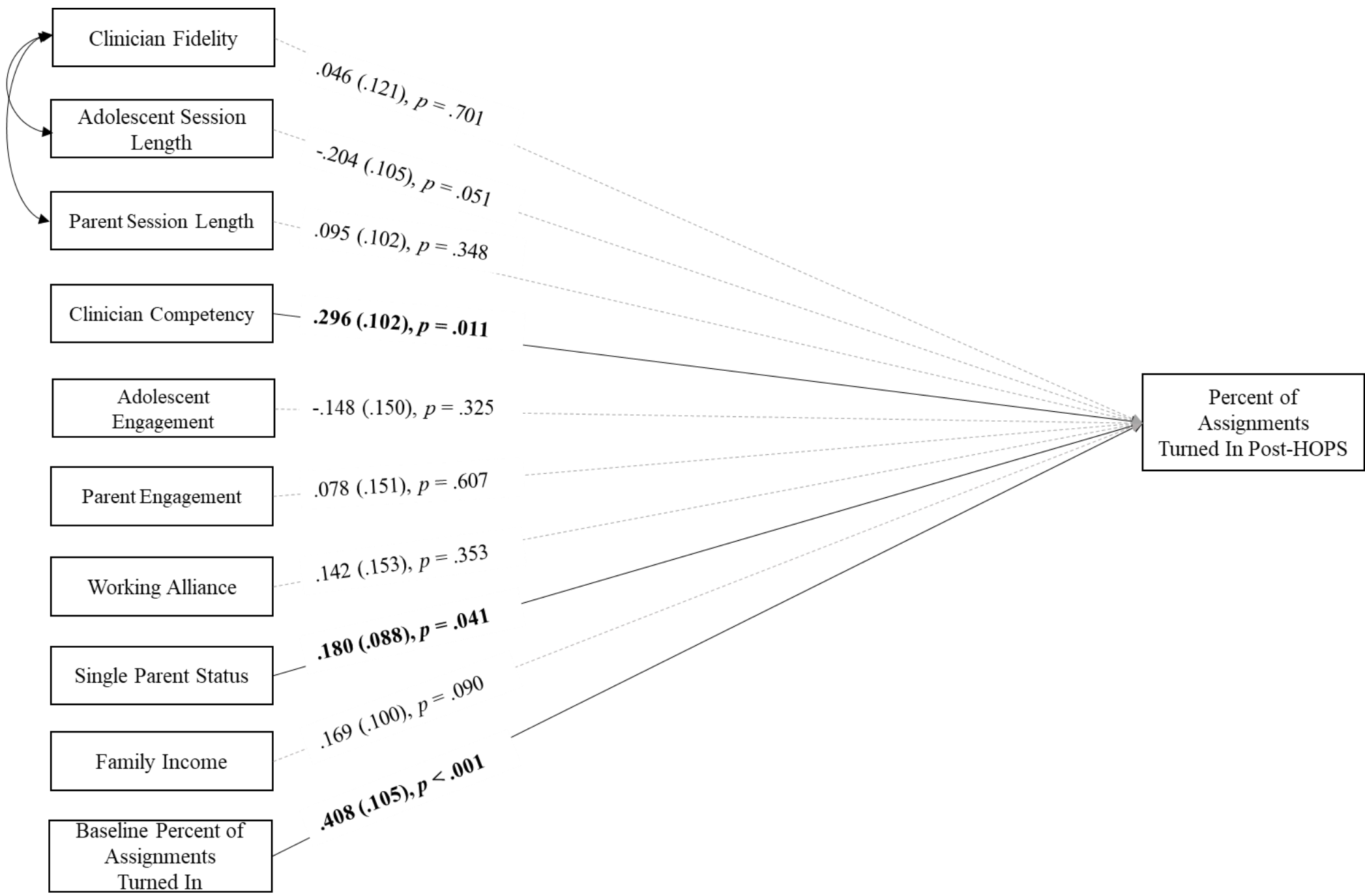

Note. $\mathrm{ADHD}=$ attention-deficit/hyperactivity disorder; HOPS = Homework, Organization, and Planning Skills. Standardized coefficients presented in figure. 